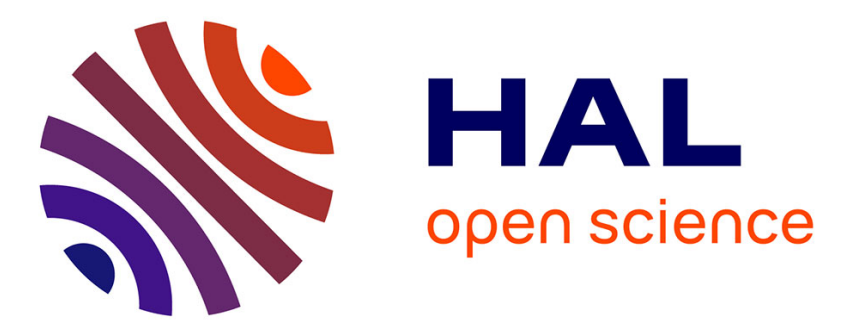

\title{
Radiative properties of silica nanoporous matrices
}

Sylvain Lallich, Franck Enguehard, Dominique Baillis

\section{To cite this version:}

Sylvain Lallich, Franck Enguehard, Dominique Baillis. Radiative properties of silica nanoporous matrices. 8th International Vacuum Insulation Symposium, Sep 2007, Würzburg, Germany. hal01290207

\section{HAL Id: hal-01290207 \\ https://hal.science/hal-01290207}

Submitted on 17 Mar 2016

HAL is a multi-disciplinary open access archive for the deposit and dissemination of scientific research documents, whether they are published or not. The documents may come from teaching and research institutions in France or abroad, or from public or private research centers.
L'archive ouverte pluridisciplinaire HAL, est destinée au dépôt et à la diffusion de documents scientifiques de niveau recherche, publiés ou non, émanant des établissements d'enseignement et de recherche français ou étrangers, des laboratoires publics ou privés. 


\title{
Radiative properties of silica nanoporous matrices
}

\author{
Sylvain LALLICH ${ }^{1,2}$, Franck ENGUEHARD ${ }^{1 *}$ and Dominique BAILLIS ${ }^{2}$ \\ ${ }^{1}$ CEA / Le Ripault, BP 16, F-37260 Monts, France \\ ${ }^{2}$ CETHIL, UMR5008, CNRS, INSA-Lyon, Université Lyon1, F-69621, Villeurbanne, FRANCE \\ *(Corresponding author : franck.enguehard@cea.fr)
}

\section{Introduction}

Nanoporous superinsulating materials are currently the subject of much attention because of their awesome thermal insulation properties: up to five times better than air, generally regarded as an excellent thermal insulator, when they are placed under primary vacuum. These materials are made of nanoporous matrices of amorphous silica nanoparticles, fibres to provide mechanical reinforcement and micrometric particles to improve opaqueness in the infrared wavelength region. Our aim is to determine experimentally and to model the radiative properties of such nanoporous materials, which are semi-transparent media in the considered wavelength range. In the simplified approach presented here, we consider the nanoporous matrix alone.

We first present the characteristics of the samples and the way we used to get their optical properties. Then after a brief review of the radiative transfer equation (RTE) and of the parameter identification technique used, we discuss the radiative properties spectra obtained. Lastly, the radiative properties computed using two different theoretical methods are compared to the experimentally determined spectra.

\section{Determination of optical properties of nanoporous matrices}

The studied material is a silica nanoporous matrix obtained by packing pyrogenic silica nanoparticles supplied by Wacker (HDK-T30). Using TEM images, the diameter of the primary nanoparticles has been estimated between 10 and $15 \mathrm{~nm}$. The samples obtained are highly porous (90\%) and have different thickness's between 2.0 and $10.5 \mathrm{~mm}$. All these samples are optically characterized using two different spectrometers covering an overall spectral band of [250 nm; $20 \mu \mathrm{m}$ ]. In order to measure the hemispherical transmittance and reflectance properties, the two spectrometers are equipped with integrating spheres that collect hemispherically the radiation travelling through or reflected by the samples.

Figure 1 shows the spectra obtained for three samples of thickness $2.8 \mathrm{~mm}, 5.9 \mathrm{~mm}$ and $10.5 \mathrm{~mm}$. These spectra are in accordance with what we expect. As far as the transmittance is concerned, we can point out that it decreases as the thickness of the sample increases. In the neighbourhood of $3 \mu \mathrm{m}$ wavelength, the transmittance almost nullifies: this large absorption peak is attributed to the presence of water. Above $8 \mu \mathrm{m}$, the transmittance is nil because of the absorption of silica that strongly increases in this wavelength range. With regard to the reflectance, it increases with the thickness of the sample: this behaviour is explained by the fact that the quantity of backscattered photons increases with the sample thickness. Above $8 \mu \mathrm{m}$, the reflectance doesn't depend anymore on the thickness of the sample; as the silica is very absorbing in this wavelength range, even the thinnest 
sample can be considered as optically thick.

\section{Determination of radiative properties of nanoporous matrices}

Once the optical properties of the samples are determined, we have to translate this data into radiative properties. To this end, we use the Radiative Transfer Equation (RTE) formalism and a parameter identification technique.

First, we consider the sample as a homogeneous medium. Moreover, we assume one-dimensional radiative transfer with azimuthal symmetry. Lastly, we ignore the self-emission term. Under these assumptions, we can write the RTE as follow:

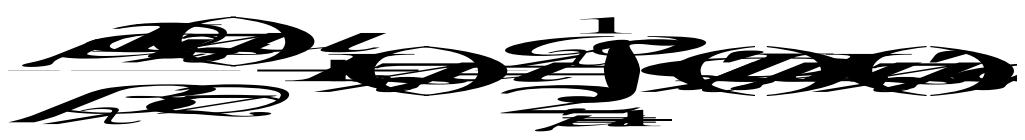

where $L_{\lambda}$ is the intensity, $z$ is the spatial coordinate (liing between 0 and the thickness $t$ of the sample), $\beta_{\lambda}$ the monochromatic extinction coefficient, $\omega_{\lambda}$ the monochromatic scattering albedo, $\Phi_{\lambda}$ the spectral scattering phase function, and $\mu$ the cosine of the polar angle.

Since the diameter of the nanoparticles constituting the material is close to $10 \mathrm{~nm}$ and the wavelength considered are of the order of $1 \mu \mathrm{m}$, we clearly lie in the Rayleigh regime. So we choose to use the Rayleigh phase function.

The material being very porous ( $90 \%$ ), the optical constant of the homogeneous material is close to that of air, so we neglect the reflection at the interfaces. The boundary conditions are the following:

- $\mathrm{L}_{\lambda}(\mathrm{z}=0, \mu>0)=\Psi_{\lambda}(0, \mu)$, where $\Psi_{\lambda}$ is the illumination.

- $\mathrm{L}_{\lambda}(\mathrm{z}=t, \mu<0)=0$.

As the thickness $t$ of the sample and the phase function are known, if we know $\beta_{\lambda}$ and $\omega_{\lambda}$, we can solve the problem and compute the intensity field. From that field, the hemispherical transmittance and reflectance express:

$$
T_{\lambda}^{h}=\frac{\int_{0}^{1} L_{\lambda}(t, \mu) \mu d \mu}{\int_{0}^{1} \Psi_{\lambda}(0, \mu) \mu d \mu} \quad R_{\lambda}^{h}=\frac{\int_{-1}^{0} L_{\lambda}(0, \mu) \mu d \mu}{\int_{0}^{1} \Psi_{\lambda}(0, \mu) \mu d \mu}
$$

The intensity field is computed using the Discrete Ordinates Method (DOM) that consists in replacing the angular integrals with quadrature:

$$
\int_{4 \pi} f(\vec{\Delta}) d \Omega \approx \sum_{i=1}^{N} w_{i} f\left(\Delta_{i}\right)
$$

Using the DOM, the new form of the RTE is:

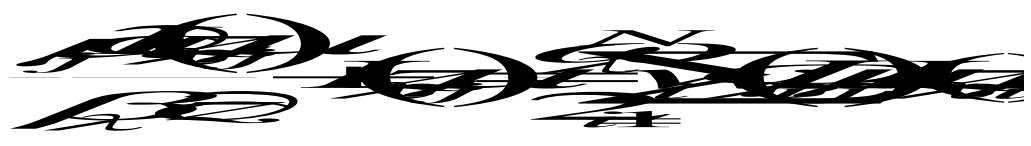


In the same way, the hemispherical transmittance and reflectance become:
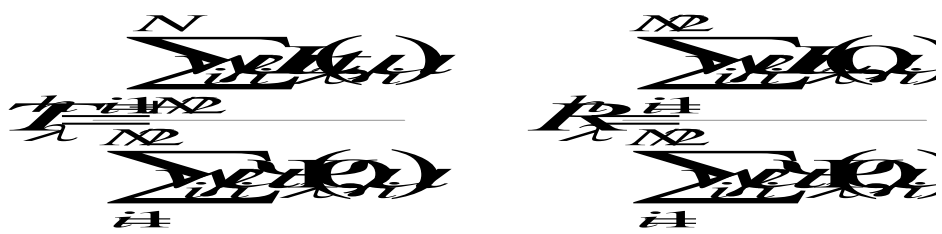

All this procedure can be considered as the direct problem, namely obtaining the optical properties of our samples from their radiative properties. So we inverse the problem using a method based on the Newton-Raphson algorithm. The radiative propertie spectra obtained that way are shown in figure 2 and 3.

\section{$4 \quad$ Comparison between experimentally determined and theoretical radiative properties}

One of the main goals of the work is to predict the radiative behaviour of the nanostructures we are interested in. In a first time, we try to compare the experimentally determined radiative properties to the radiative properties computed with the Mie theory. In a second time, due to the non-expected results obtained with the Mie theory, we try a method based on the Discrete Dipole Approximation (DDA).

\subsection{Comparison of the radiative properties experimentally determined and the Mie theory}

As the studied material is made of packed spherical nanoparticles, we first try to use the Mie theory [Van de Hulst, 1957] to predict radiative properties of our samples. The Mie theory is used to compute the interaction (scattering and absorption) between a sphere and an electromagnetic wave.

As the pyrogenic silica used to make the samples is very hydrophilic, to take into account the water contribution, we use the coated sphere model developed by [Borhen et al., 1983]. We choose a thickness of coating that sounds realistic: the same order as the diameter of a water molecule. Assuming $10 \mathrm{~nm}$ diameter particles, the radiative properties we obtain are far from the experimentally determined ones, notably in the wavelength range in which scattering dominates the radiative transfer (figure 4). The only way that we found to get a good agreement on the whole wavelength range considered is to increase the particle diameter up to $55 \mathrm{~nm}$ (figure 5). Consequently from the point of view of the interaction with electromagnetic waves, our materials behaves approximately as a cloud of uniform size particles of about $55 \mathrm{~nm}$ diameter. Although this value is significantly different from the diameter of the primary particles, it is not an absurd value: indeed during the silica powder fabrication process the primary nanoparticules of $10 \mathrm{~nm}$ diameter fuse together to form larger units of approximately $120 \mathrm{~nm}$ hydrodynamic equivalent-sphere diameter [Wacker]. If we assume that the porosity of these aggregates is the same as the one of the matrix, the volume of silica contained in such an aggregate is equivalent to the volume of a dense silica sphere of $55 \mathrm{~nm}$ diameter. Hence, we can venture the hypothesis that the nanoporous matrix scatters as a cloud of primary scatterers, the primary scatterer being here the aggregate obtained during the production process.

In order to confirm this hypothesis, we use a second way to compute the radiative properties of the samples that takes into consideration the microstructure: the Discrete Dipole Approximation.

\subsection{Comparison of the radiative properties experimentally determined and}




\section{the Discrete Dipole Approximation}

The DDA [Draine et al., 1994] is a flexible method that allows to compute the absorption and scattering properties of irregular targets (particles of complex shapes, clusters of spheres) approximated by arrays of point dipoles. The DDA calculations require a detailed description of the scatterer he localization of the dipoles in space and the evaluation of their polarizabilities. Concerning the polarizabilities, since we apply the DDA on a cluster of spherical particles that are small compared to the wavelength, we may treat each particle as a single dipole using the $a_{1}$-term method ${ }^{6}$. This method has been shown to be superior to the other polarizability prescriptions for a cluster of spherical monomers replaced by single dipoles [Okamoto, 1995]. The dipole moments within the cluster result on the one hand from an incident electromagnetic field that activates them, and on the other hand from the interaction between them; once these dipole moments are solved, it is possible to determine the radiative properties of the cluster.

Prior to DDA calculations, the dipoles must be localized in space. To obtain valuable results, the material structures on which DDA calculations are performed must be representative of our nanoporous material in terms of porosity $(\approx 90 \%)$, specific surface area $\left(\approx 250 \mathrm{~m}^{2} \cdot \mathrm{gr}^{-1}\right)$ and fractal dimension $(\approx 1.8$ according to the litterature [Legrand, 1998]). To this end, we have generated numerical structures with the help of the Diffusion Limited Cluster-Cluster Aggregation algorithm [Kolb et al., 1983] [Meakin, 1983]. We have integrated the water contribution in the relative dielectric permittivity function and hence in the polarisability function of the particles with the help of the Maxwell-Garnett mixing rule. We have generated clusters of 170 particles of $10 \mathrm{~nm}$ diameter, coated with $2 \AA$ of water. The number of 170 particles was chosen in order to form clusters of $120 \mathrm{~nm}$ equivalent diameter and of $90 \%$ porosity. The satisfactory correspondence between the experimental and simulated radiative properties spectra is quite encouraging, and is a first step toward a reasonable representation of the material organization within our nanoporous matrices.

\section{$5 \quad$ Conclusion and outlook}

We have determined the spectral radiative properties of silica nanoporous matrices from experimentally obtained reflectances and transmittances using a parameter identification. These data have been compared to the Mie theory assuming a uniform diameter distribution of $10 \mathrm{~nm}$ for the particles. To get a good agreement between the experimental values and the Mie theory, we had to increase the particle diameter up to $55 \mathrm{~nm}$.

From the very little we know about the silica powder, we have proposed an explanation that seems to be confirmed by the first DDA calculations. Nonetheless, these results must be toned down because they are based on the use of a structure with a given fractal dimension, that is close to what is usually found in the literature. Nevertheless, as many other different fractal dimension values can be found, depending notably on the particle size and volume fraction, fractal dimension measurements of our samples are in progress using $\mathrm{X}$-ray and neutron scattering techniques. 


\section{References:}

[Van de Hulst, 1957] Light scattering by small particles. John Wiley \& Sons, New York, 1957

[Bohren et al., 1983] Absorption and scattering of light by small particles. John Wiley and Sons, NewYork, 1983

[Yurkin et al., 2007] The discrete dipole approximation: An overview and recent developments. Journal of Quantitative Spectroscopy and Radiative Transfer (2007), doi:10.1016/j.jqsrt.2007.01.034

[Kolb et al., 1983] Scaling of kinetic growing cluster. Physical Review Letters, 51(13):1123, 1983

[Meakin, 1983] Formation of fractal clusters and networks by irreversible diffusionlimited aggregation. Physical Review Letters, 51(13):1119, 1983

[Legrand, 1998] The surface properties of silicas. JohnWiley \& Sons, 1998

[Draine et al., 1994] Discrete dipole approximation for scattering calculations, Journal of the Optical Society of America A, 11(4):1491,1994

[Okamoto, 1995] Light scattering by clusters : the a1-term method, Optical Review, 2(6):407, 1995

[Wacker] http://www.wacker.com/internet/webcache/de_DE/_Downloads/CMP_en.pdf http://www.wacker.com/internet/webcache/de_DE/_Downloads/Basis_en.pdf 

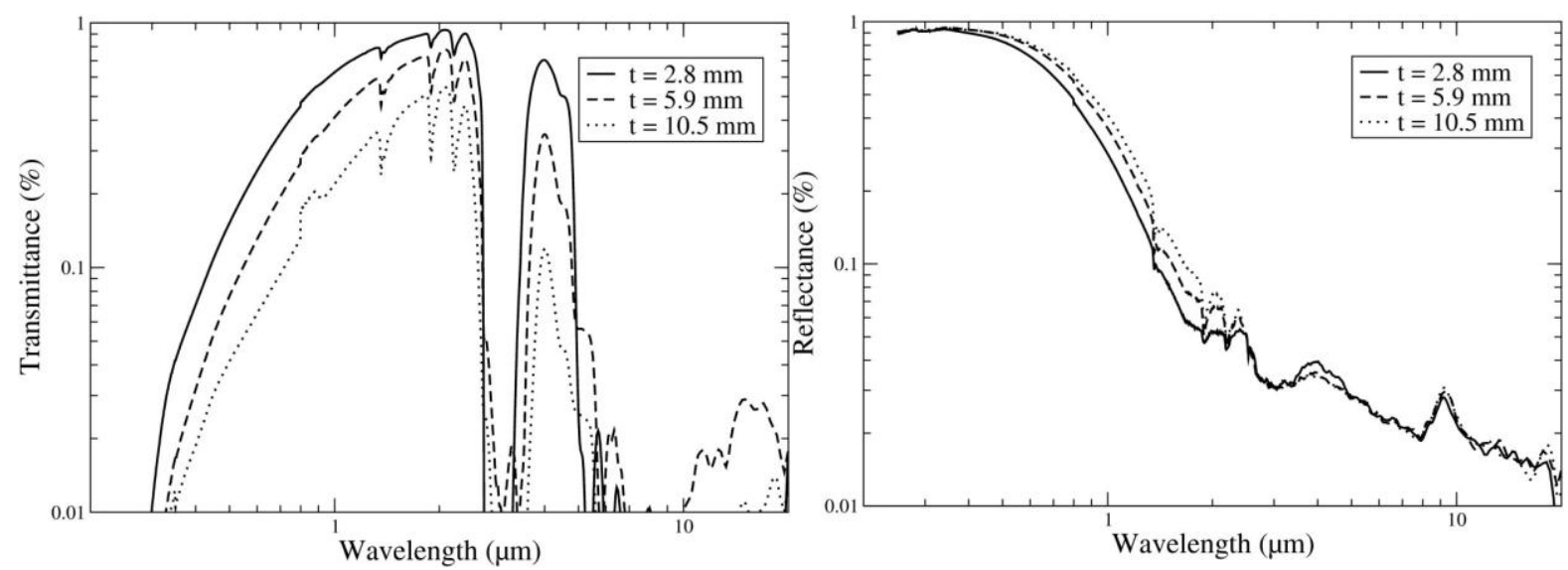

Figure 1: Hemispherical transmittance and reflectance spectra in the spectral band [250 $\mathrm{nm} ; 20 \mu \mathrm{m}]$ for three samples of different thickness.
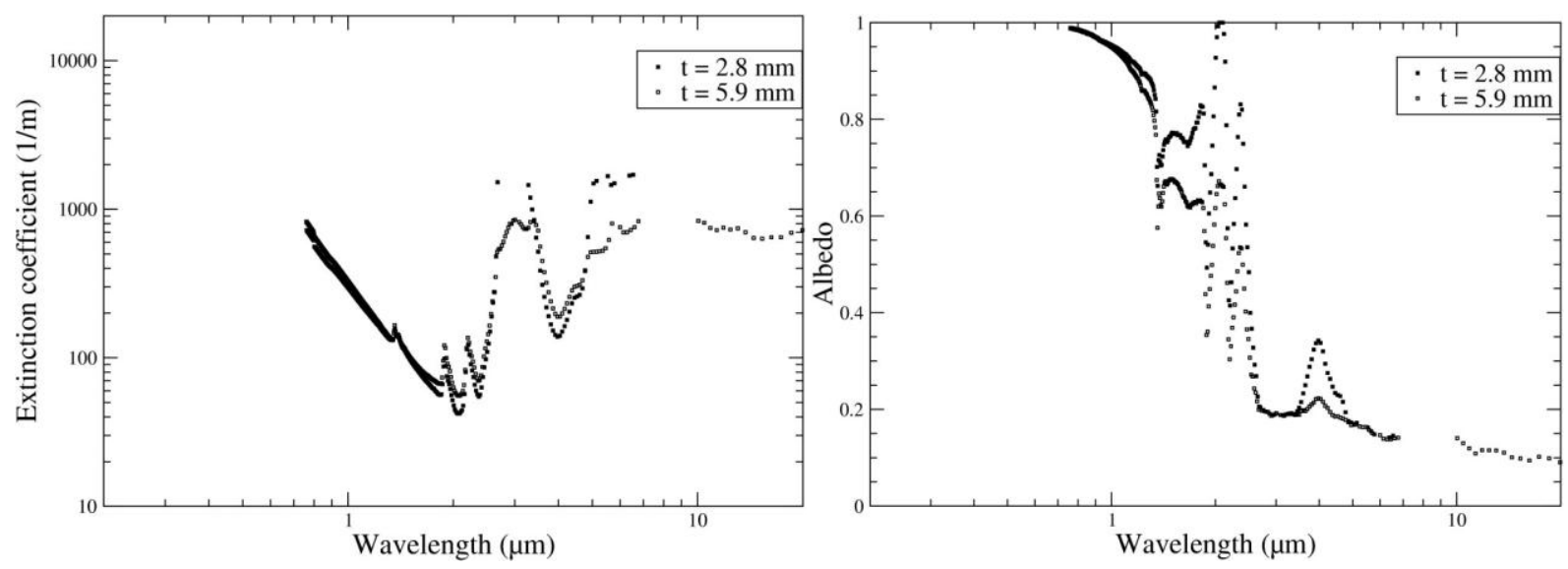

Figure 2 : Extinction coefficient and albedo of two samples of different thickness obtained by parameter identification (measurement in November 2005) 

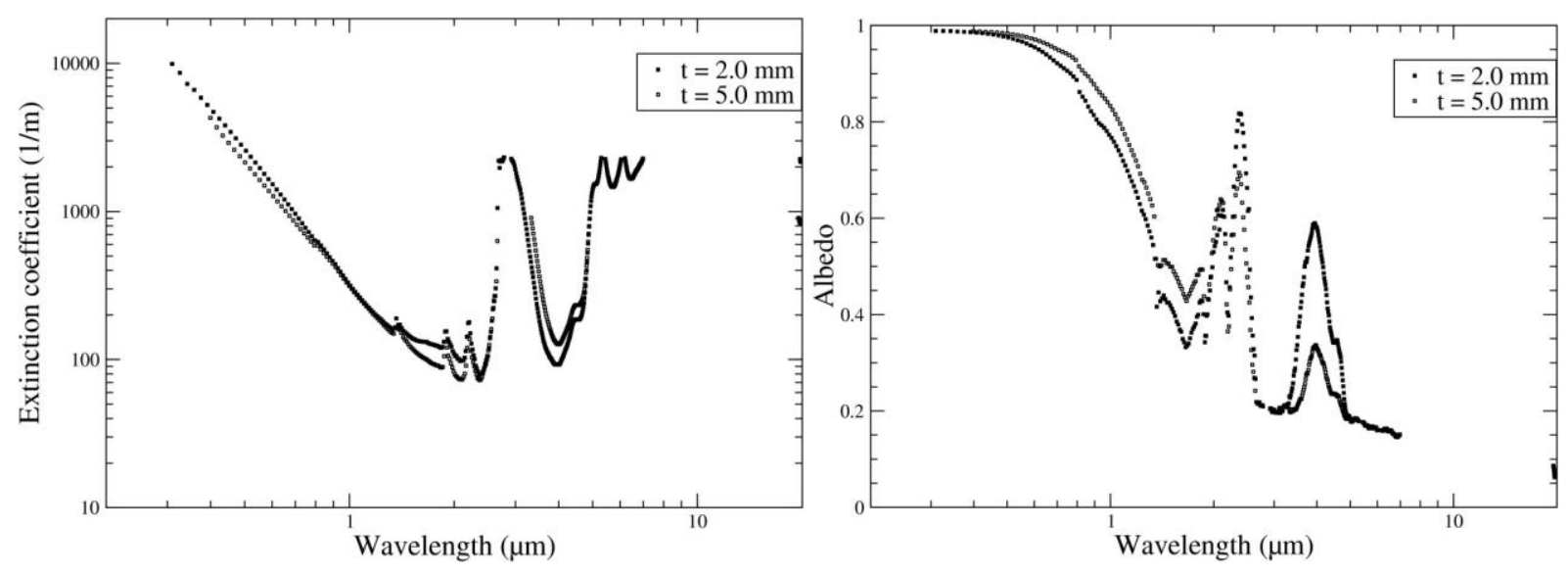

Figure 3 : Extinction coefficient and albedo of two samples of different thickness obtained by parameter identification (measurement in December 2006)
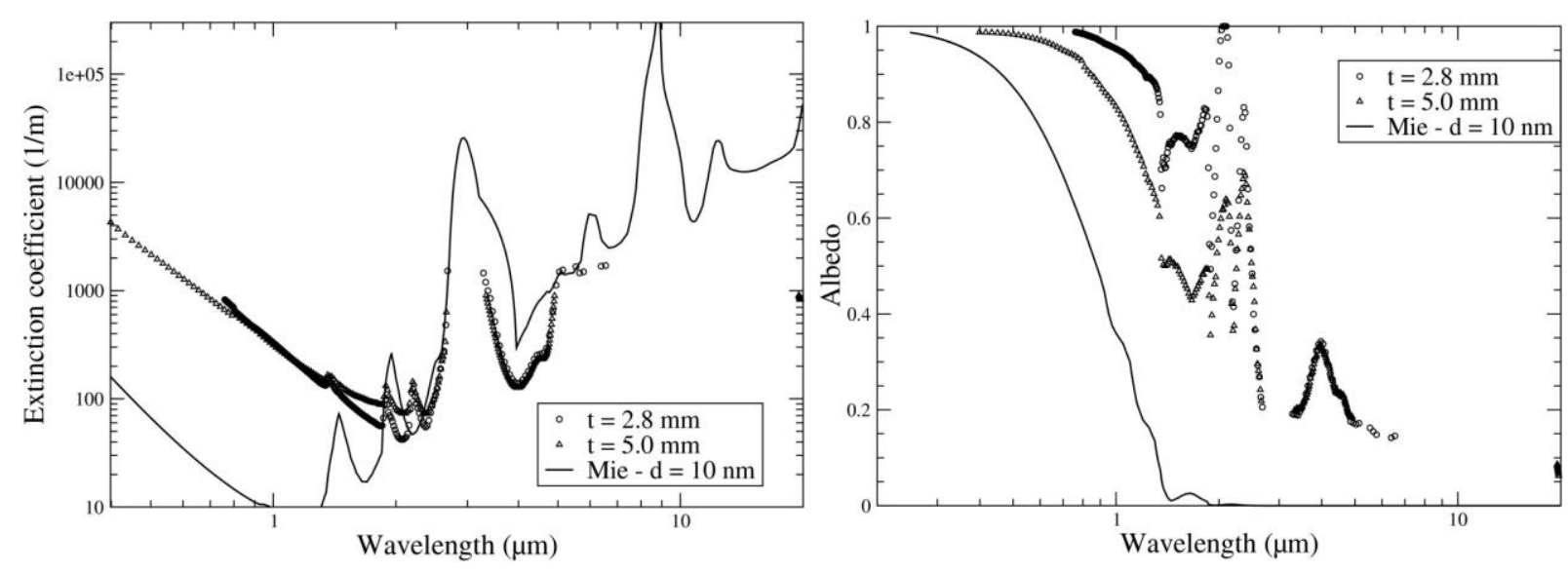

Figure 4 : Comparison of the extinction coefficient and the albedo between two different experimental results and the Mie theory. The parameters used for the Mie calculation are $\mathbf{1 0} \mathbf{~ n m ~ d i a m e t e r ~}$ nanoparticules with a 2 A thick coating 

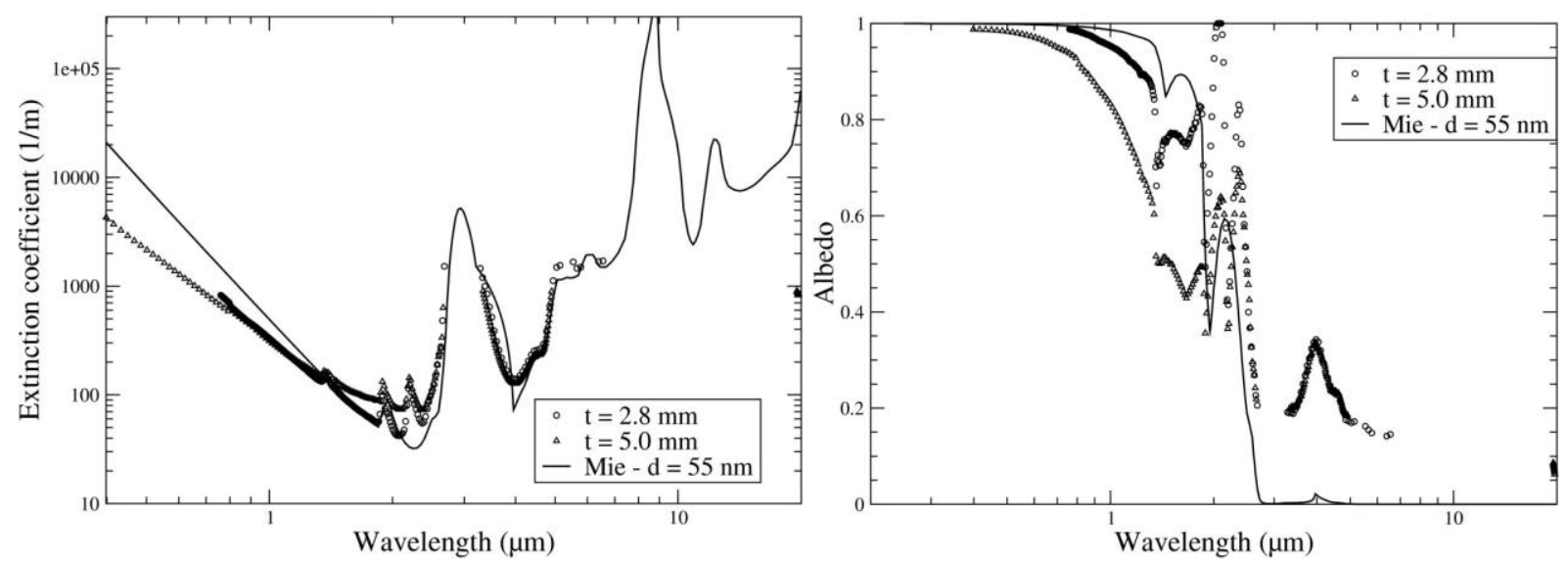

Figure 5 : Comparison of the extinction coefficient and the albedo between two different experimental results and the Mie theory. The parameters used for the Mie calculation are $55 \mathbf{~ n m}$ diameter nanoparticules with a 2 thick coating
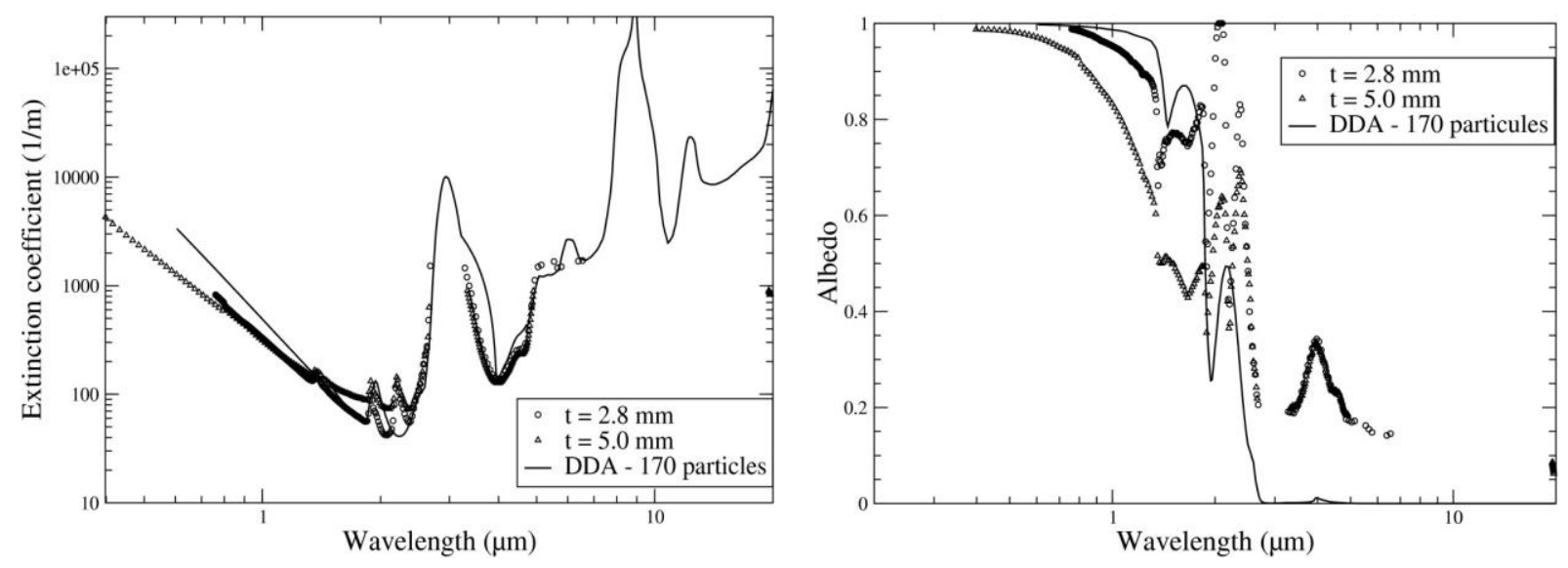

Figure 6 : Comparison of the extinction coefficient and the albedo between two different experimental results and the Discrete Dipole Approximation. For the DDA calculation, we assume $10 \mathrm{~nm}$ diameter spherical nanoparticles that don't overlap. The water contribution is taken into account using a Maxwell-Garnett mixing rule on the relative dielectric function. 